\title{
Norepinephrine Regulates Keratinocyte Proliferation to Promote the Growth of Hair Follicles
}

\author{
Yanan Konga,b Yishu Liu b, e Liangli Pan ${ }^{\mathrm{a}, \mathrm{b}}$ Biao Cheng ${ }^{\mathrm{a}-c, \mathrm{e}}$ Hongwei Liu ${ }^{\mathrm{d}}$

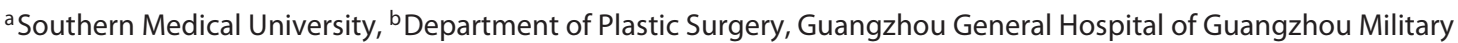 \\ Command, PLA, ${ }^{C}$ Key Laboratory of Trauma Treatment and Tissue Repair of Tropical Area, PLA, and ${ }^{\mathrm{d}}$ Department of \\ Plastic Surgery, The First Affiliated Hospital of Jinan University, Guangzhou, and ${ }^{\mathrm{e}}$ Third Military Medical University, \\ Chongqing, PR China
}

\section{Key Words}

Sympathetic nerve $\cdot$ Hair follicle $\cdot$ Norepinephrine $\cdot$

Propranolol · Keratinocyte

\section{Abstract}

Psychological factors and stress can cause hair loss. The sympathetic-adrenal-medullary (SAM) axis has been reported to regulate the growth of hair follicles (HF). The sympathetic nerve is a component of the SAM axis, but it has not been sufficiently or convincingly linked to hair growth. In this study, we demonstrate that chemical sympathectomy via administration of the neurotoxin 6-hydroxydopamine (6OHDA) to mice inhibited HF growth, but treatment with the $\beta$-adrenoceptor antagonist propranolol (PR) had no effect. $\mathrm{HF}$ length and skin thickness were greater in PR-treated and control mice than in 6-OHDA-treated mice, as evidenced by hematoxylin and eosin staining. Furthermore, we found that the reduced HF growth in sympathectomized animals was accompanied by a decreased keratinocyte proliferation. Moreover, the neurotransmitter norepinephrine (NE) was found to efficiently promote HF growth in an organotypic skin culture model. Together, these findings suggest that

\section{KARGER}

(C) 2016 S. Karger AG, Basel

$1422-6405 / 16 / 2016-0423 \$ 39.50 / 0$

E-Mail karger@karger.com

www.karger.com/cto sympathetic nerves regulate keratinocyte behaviors to promote hair growth, providing novel insights into stress-related, chemotherapy-, and radiotherapy-induced alopecia.

(c) 2016 S. Karger AG, Basel

\section{Introduction}

Psychological factors have been demonstrated to cause hair loss [Wygledowska-Kania and Bogdanowski, 1996; Trueb, 2010]. Data obtained from animal models support the hypothesis that stress may act as a cofactor to promote hair loss [Peters et al., 2006]. The sympathetic-adrenalmedullary (SAM) and hypothalamic-pituitary-adrenal axes are activated by higher brain centers in response to psychological stress [Arck et al., 2006; Slominski, 2007; Heidt et al., 2014].

The skin is a neuroendocrine organ that is a target of hormones, neurohormones, neurotransmitters, and neu-

Biao Cheng

Department of Plastic Surgery

Guangzhou General Hospital of Guangzhou Military Command, PLA

Liuhua Road 111, Guangzhou, Guangdong Province 510010 (PR China)

E-Mail chengbiaocheng@163.com

Hongwei Liu

Department of Plastic Surgery, The First Affiliated Hospital of Jinan University Huangpu Road West 613

Guangzhou, Guangdong Province 510630 (PR China)

E-Maillhw78535@163.com 


\begin{tabular}{ll}
\hline Abbreviations used in this paper \\
\hline 6-OHDA & 6-hydroxydopamine \\
AR & adrenoreceptor \\
A-SC & activated stem cells \\
BrdU & bromodeoxyuridine \\
Bu-SC & stem cells located in the bulge \\
CRH & corticotropin-releasing hormone \\
CRH-R1 & corticotropin-releasing hormone receptor-1 \\
CTR & control \\
DP & dermal papilla \\
FGF & fibroblast growth factor \\
HE & hematoxylin and eosin \\
HF & hair follicle \\
HG & hair germ \\
IFE & interfollicular epidermis \\
i.p. & intraperitoneal \\
NE & norepinephrine \\
PBS & phosphate-buffered solution \\
PH & phentolamine \\
POMC & proopiomelanocortin \\
PR & propranolol \\
SAM & sympathetic-adrenal-medullary \\
SC & stem cells \\
TAC & transit-amplifying cells \\
\hline &
\end{tabular}

ral responses [Slominski et al., 2007]. The skin is also regulated by the SAM and hypothalamic-pituitary-adrenal axes and expresses elements of these axes, including proopiomelanocortin (POMC), corticotropin-releasing hormone (CRH), CRH receptor-1 (CRH-R1), and adrenoreceptors (AR) [Slominski and Wortsman, 2000; Slominski et al., 2007]. Stress-induced signals induce neurons to produce and release $\mathrm{CRH}$. CRH then binds $\mathrm{CRH}-\mathrm{R} 1$ and induces the release of POMC-derived adrenocorticotropic hormone, which stimulates glucocorticoid synthesis and secretion [Slominski and Wortsman, 2000; Slominski et al., 2000]. Activation of the SAM axis increases the release of the neurotransmitters epinephrine and norepinephrine (NE) from the adrenal medulla and stimulates the sympathetic norepinergic nerves to promote further NE secretion [Glaser and Kiecolt-Glaser, 2005; StenerVictorin et al., 2005]. Past studies have focused on regulation of the skin by the hypothalamic-pituitary-adrenal axis. However, little is known about the SAM axis and specifically the sympathetic nerve.

Mammalian skin is composed of a pilosebaceous unit containing a hair follicle (HF) and its surrounding interfollicular epidermis (IFE) which together maintain skin homeostasis. Cells located in the IFE proliferate, migrate, and perform skin barrier functions. HF contain sebaceous glands that are formed from and replenished by resident stem cells (SC) located in the outer root sheath. Following HF degeneration, the resident SC differentiate into sebocytes, which release oils that lubricate the hair channel and skin surface [Blanpain and Fuchs, 2009; Slominski et al., 2012].

As a component of the SAM axis, the sympathetic nerve can regulate wound healing by secreting neurotransmitters such as NE, a key mediator of cell proliferation, differentiation, and apoptosis [Yang et al., 2006]. Sympathetic nerve fibers are located between the blood vessels and HF and insert into the HF outer root sheath at the level of the bulge region, the probable reservoir of HF SC. In addition, some circumstantial evidence supports the involvement of sympathetic skin nerves in HF growth [Asada-Kubota, 1995; Peters et al., 2007]

$\mathrm{HF}$ are circulating growth organs whose cyclic transformations consist of phases of growth (anagen), regression (catagen), and rest (telogen) [Peters et al., 2006; Ito, 2010; Obeid et al., 2013]. The HF cyclic process is characterized by dramatic changes in skin physiology, with variations in $\mathrm{CRH}$ content, expression of $P O M C$, production of POMC peptides, and expression of CRH-R1 and AR [Slominski et al., 1998, 2005, 2012]. Unlike the stages of the HF cycle in other mice, those in C57BL/6NCrlBR (C57BL/6) mice are macroscopically recognizable by a switch in skin color from pink to black/pink. Therefore, $\mathrm{HF}$ in C57BL/6 mice provide an ideal model system in which to examine the interactions between sympathetic nerves and the HF cycle [Muller-Rover et al., 2001].

In previous reports, mouse keratinocytes and fibroblasts have been grafted onto the dorsal skin of nude mice using chamber, flap, or patch assays. Primary keratinocytes or fibroblasts cultured alone have failed to develop $\mathrm{HF}$, but the combination of the two cell types has resulted in HF formation [Ouji et al., 2006; Liang et al., 2011]. In vivo, hair matrix keratinocytes proliferate when $\mathrm{HF}$ are in the anagen stage, whereas cells undergo apoptosis during the catagen stage [Muller-Rover et al., 2001; Blanpain and Fuchs, 2009]. Thus, normal hair growth requires a balance between keratinocyte proliferation and differentiation [Muller-Rover et al., 2001; Semenova et al., 2008; Miyata et al., 2014].

In the present study, we hypothesized that the sympathetic nervous system regulates HF growth by modulating hair matrix keratinocyte proliferation. Using C57BL/6 mice, we administered the neurotoxin 6-hydroxydopamine (6-OHDA) to selectively destroy noradrenergic nerve fiber endings as a chemically induced model of sympathectomy. We also administered the $\beta$-AR antagonist propranolol (PR) to test the effect of $\beta$-AR stimula- 
tion on HF growth. Furthermore, we assessed keratinocyte proliferation using bromodeoxyuridine (BrdU) and Ki67 staining and developed an organotypic skin culture model to examine the role of NE on HF formation.

\section{Materials and Methods}

Animals

Ninety 4-week-old female C57BL/6 mice were purchased from the Guangdong Medical Laboratory Animal Center. The mice were housed and handled strictly in accordance with the recommendations of the Guide for the Care and Use of Laboratory Animals of the National Institutes of Health. All protocols were approved by the Bioethics Committee of the General Hospital of Guangzhou Military Command. Mice were randomly assigned to the following 3 groups in a 1:1:1 ratio: 6-OHDA, PR, and control (CTR) ( $\mathrm{n}=30$ /group). All efforts were made to minimize suffering. We did not conduct research outside of our country of residence [Jia et al., 2013].

\section{Pharmacological Modulation of Sympathetic Nervous System} Signals

PR (0.5 g/l; Sigma, Buchs, Switzerland) was administered into the drinking water of the mice in the PR group, whereas pure water was provided to the mice in the 6-OHDA and CTR groups. After 2 weeks, 6-week-old mice in the 6-OHDA group received intraperitoneal (i.p.) injections of 6-OHDA (Sigma) diluted in a sterile saline solution containing $0.01 \% \mathrm{~L}$-ascorbic acid. A dose of 100 $\mathrm{mg} / \mathrm{kg}$ of 6-OHDA was administered on the first day and $250 \mathrm{mg} /$ $\mathrm{kg}$ was given on subsequent days. 6-OHDA was injected at 8.00 a.m. and 8.00 p.m. on the day of administration every 2 days for 3 weeks. Six-week-old mice in the PR and CTR groups received i.p. injections of equal amounts of solvent (sterile saline solution containing $0.01 \% \mathrm{~L}$-ascorbic acid).

\section{Depilation}

Depilation was conducted on the seventh day of 6-OHDA or solvent administration in mice (7 weeks old) over $20 \mathrm{~g}$, as previously described [Gosain et al., 2006; Katayama et al., 2006]. Based on body weight, 28 mice were included in the 6-OHDA group, 28 in the PR group, and 30 in the CTR group. Mice were anesthetized via i.p. injection of $10 \%$ chloral hydrate (w/v, $0.002 \mathrm{ml} / \mathrm{g}$ of body mass; Sigma, St. Louis, Mo., USA) [Jia et al., 2013]. The back skin was shaved (area: $3 \times 2.5 \mathrm{~cm}$ ) and a depilatory cream (Veet, London, UK) was applied to areas where all HF were in the telogen stage for $3 \mathrm{~min}$, as evidenced by homogeneous pink-colored skin [Jia et al., 2013]. Following the removal of the cream with humid gauze, the HF immediately and uniformly entered the anagen stage. A gray color was defined as the maker of the anagen stage [Muller-Rover et al., 2001].

\section{Skin Harvesting}

Skin was harvested on days 1, 3, 5, 7, 9, and 14 after depilation. To detect HF epithelial proliferation, mice received i.p. injections of BrdU ( $0.2 \mathrm{mg} / \mathrm{g}$ of body mass; Sigma) $2 \mathrm{~h}$ before harvesting of the skin, as previously described [Zhang et al., 2011]. Mice were sacrificed via i.p. injection of a lethal dose of chloral hydrate. Two mice per group at a time were chosen at random and sacrificed.
The back skin from the neck region was harvested perpendicularly to the paravertebral line to obtain longitudinal HF sections. The middle dorsal skin (area: $1 \times 0.5 \mathrm{~cm}$ ) was carefully dissected and fixed in $4 \%$ paraformaldehyde. Residual samples were immediately frozen in liquid nitrogen and further processed for morphometric analyses as described below [Jia et al., 2013].

\section{Hematoxylin and Eosin Staining}

The skins were dehydrated in a graded ethanol series, cleared in dimethylbenzene, and embedded in paraffin. Sections $(5 \mu \mathrm{m})$ were deparaffinized with immersion in dimethylbenzene and rehydrated. Hematoxylin and eosin (H\&E) staining was performed according to standard procedures [Jia et al., 2013]. Staining was visualized under an Olympus BX51 microscope. Three slices were obtained from each paraffin-embedded sample, and 6 slices were selected for staining within each group. Images were captured using ImageJ software, and 18 photomicrographs (magnification $\times 200$ ) were randomly selected from each group to measure the HF length and skin thickness.

HF length was measured as the distance from the dermal papilla (DP) to the epidermis. Skin thickness was measured from the surface of the epidermis to the muscle. The mean length of all HF was calculated per photomicrograph. The mean skin thickness was calculated by dividing the area of the epidermis and the subcutis by the width of the photomicrograph [Hansen et al., 1984].

\section{Immunohistochemistry}

Briefly, the sections were deparaffinized with immersion in dimethylbenzene, rehydrated, heated in citrate buffer $(0.01 \mathrm{M}, \mathrm{pH}$ 6.0) for $5 \mathrm{~min}$ at $100^{\circ} \mathrm{C}$, and then treated with endogenous peroxidase ( $3 \%$ hydrogen peroxide solution) for $5 \mathrm{~min}$ at room temperature. After blocking in 10\% goat serum for another $30 \mathrm{~min}$ at room temperature, the sections were immunostained with primary antibodies for Ki67 (1:500; Abcam, Cambridge, UK) or BrdU (1:500; Sigma) diluted in phosphate-buffered solution (PBS) containing $0.1 \%$ Tween-20 and $5 \%$ bovine serum albumin overnight at $4^{\circ} \mathrm{C}$. To detect $\mathrm{BrdU}$, the sections were incubated in $2 \mathrm{M} \mathrm{HCl}$ for $30 \mathrm{~min}$ at $37^{\circ} \mathrm{C}$ and in $0.1 \mathrm{M}$ borate buffer for $10 \mathrm{~min}$. The sections were then washed 3 times with PBST (PBS containing $0.1 \%$ Tween-20) and incubated with secondary antibodies, avidin-biotin-peroxidase complex, and DAB reagent [Zhang et al., 2011; Peng et al., 2013]. Subsequently, all sections were costained with hematoxylin and visualized under an Olympus BX51 microscope. Images were captured using ImageJ software, and 2 photomicrographs were randomly selected for each section. Positively stained cells were counted and are presented as a percentage of the total number of cells counted in the HF and in the different layers of epidermis [Cerqueira et al., 2014]. We counted $\geq 1,000$ total cells in all photomicrographs based on blue hematoxylin staining.

\section{Organotypic Skin Culture}

To investigate the potential influence of NE on the HF cycle, we cultured skin from newborn mice. The middle dorsal skin of 12 newborn mice was harvested under sterile conditions and then transferred to 12-well plates coated with gelatin (Sigma) [Botchkarev et al., 1999]. These skins were cultured in Dulbecco's modified Eagle's medium (Gibco, USA) supplemented with 10\% fetal bovine serum (Gibco), $200 \mathrm{mM} \mathrm{L-glutamine} \mathrm{(Gibco),} 100 \mathrm{U} / \mathrm{ml}$ penicillin, and $100 \mu \mathrm{g} / \mathrm{ml}$ streptomycin (Gibco) at $37^{\circ} \mathrm{C}$ under $5 \%$ $\mathrm{CO}_{2}$ and $95 \%$ air [Peters et al., 2007]. Skins were treated for 3 days 


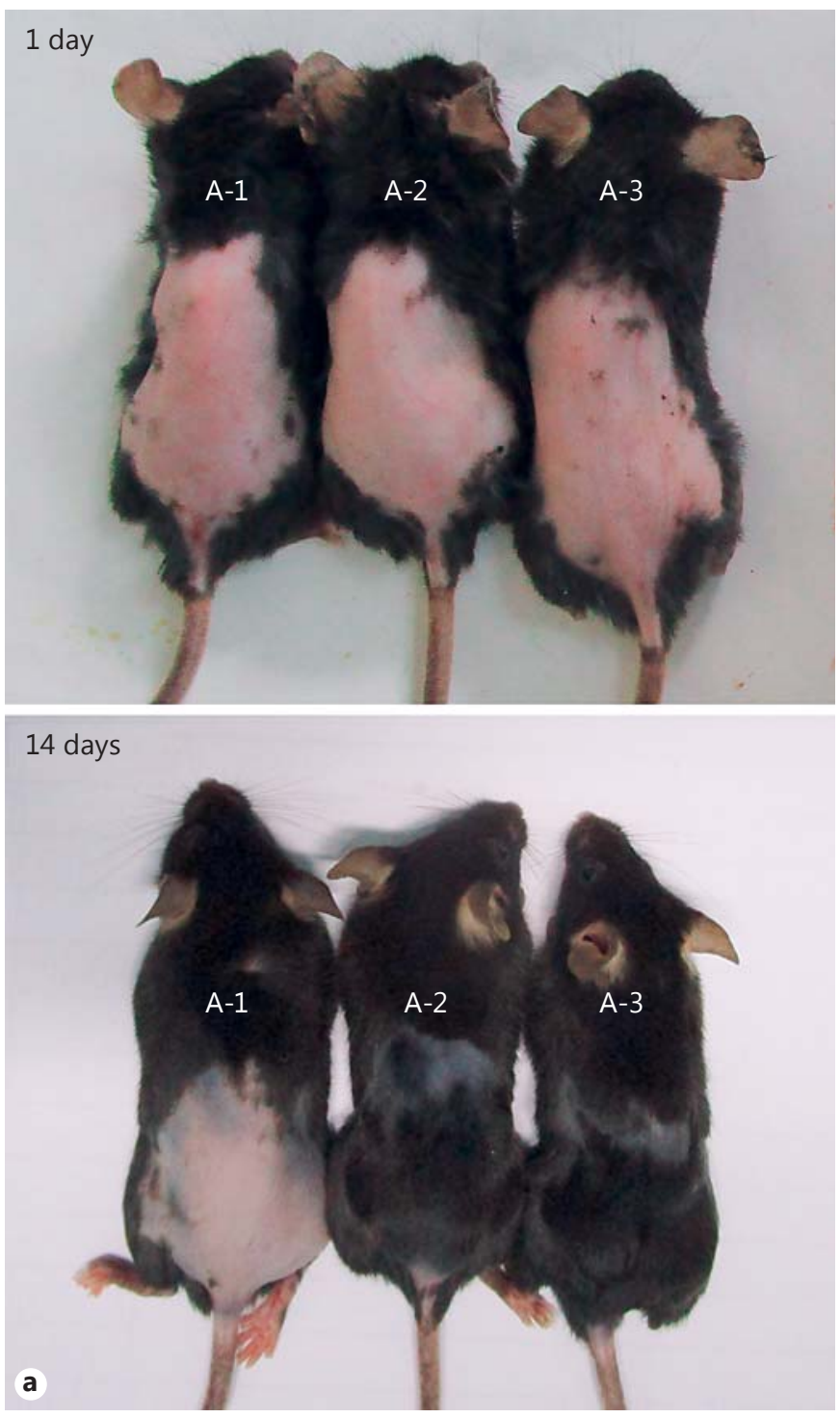

Table 1. Distribution of mice within each group over 14 days after depilation

\begin{tabular}{llrrrrrrr}
\hline \multicolumn{2}{c}{ Day: } & 1 & 3 & 5 & 7 & 9 & 11 & 14 \\
\hline 6-OHDA & $\begin{array}{l}\text { Positive } \\
\text { Total }\end{array}$ & 0 & 0 & 0 & $0^{*}$ & $2^{*}$ & $6^{*}$ & 12 \\
& 28 & 26 & 24 & 22 & 20 & 18 & 18 \\
\hline PR & Positive & 0 & 0 & 0 & 4 & 11 & 13 & 14 \\
& Total & 28 & 25 & 23 & 21 & 19 & 17 & 17 \\
\hline CTR & Positive & 0 & 0 & 0 & 6 & 13 & 15 & 16 \\
& Total & 30 & 28 & 26 & 24 & 22 & 19 & 19 \\
& & & & & & & &
\end{tabular}

Positive $=$ Mice that were gray in color ; Total $=$ total number of mice. * Statistically significant difference compared to the CTR group $(\mathrm{p}<0.05)$.

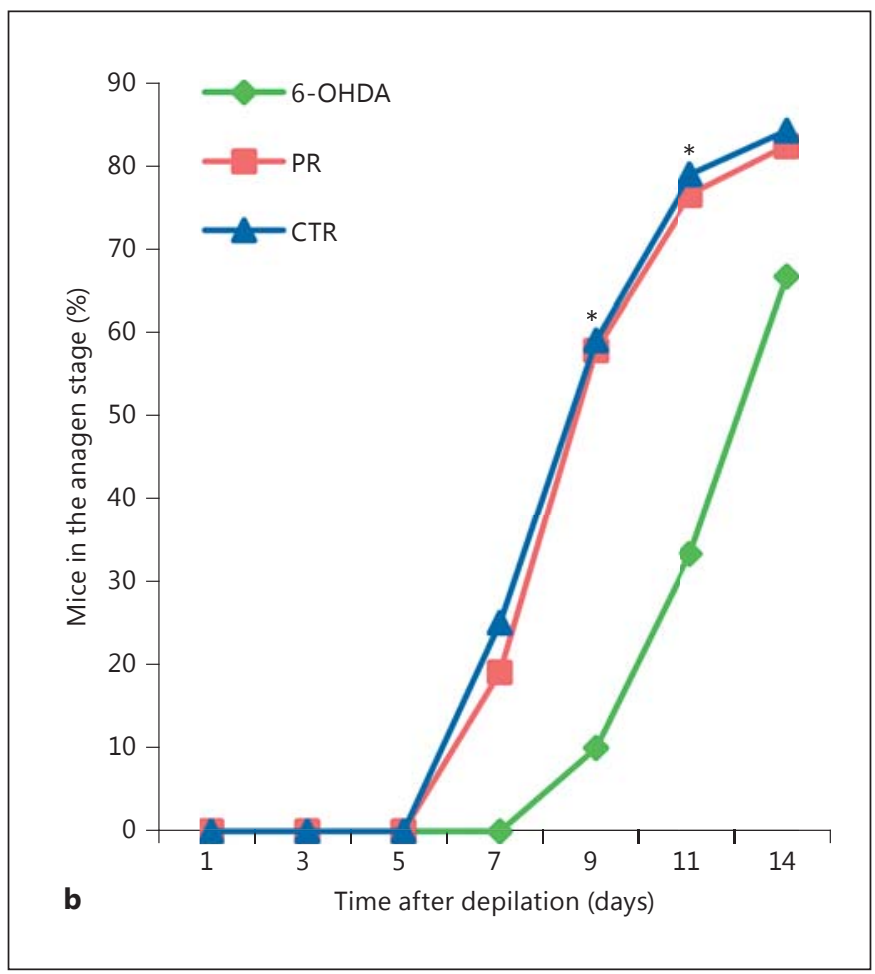

Fig. 1. Signals from the sympathetic nervous system promote HF to transition from the telogen stage to the anagen stage. Depilation was performed in mice treated with 6-OHDA (A-1) or PR (A-2) and CTR animals (A-3). a Representative images of skin color 1 and 14 days after depilation. $\mathbf{b}$ Percentage of mice exhibiting hair growth $1,3,5,7,9,11$, and 14 days after depilation. ${ }^{*} \mathrm{p}<0.05$.

with $0.1 \mathrm{M}$ PBS (pH 7.2) or $5 \mu \mathrm{M}$ NE (Sigma) and then immediately fixed in $4 \%$ paraformaldehyde for longitudinal paraffin sectioning of the HF for H\&E staining. For blocking experiments, 10 $\mu \mathrm{M}$ PR or phentolamine was added to the skin cultures for $30 \mathrm{~min}$ before the addition of $5 \mu \mathrm{M}$ NE [Yang et al., 2006]. Three slices were obtained from each paraffin-embedded sample, and 6 slices were selected for staining from each group. Images were captured using ImageJ software, and 18 photomicrographs (magnification $\times 200$ ) were randomly selected from each group to measure the HF length and skin thickness.

\section{Statistical Analysis}

Continuous variables were compared via one-way ANOVA and categorical variables via a $\chi^{2}$ test. Data were analyzed using SPSS 13.0 software (IBM SPSS) and plotted as means \pm SD. $p<0.05$ was considered statistically significant. Experiments were repeated at least 3 independent times. 
Table 2. HF length and skin thickness over 9 days after depilation

\begin{tabular}{|c|c|c|c|c|c|}
\hline \multicolumn{2}{|c|}{ Day: } & 3 & 5 & 7 & 9 \\
\hline 6-OHDA & $\begin{array}{l}\text { HF length } \\
\text { SK }\end{array}$ & $\begin{array}{c}195.79 \pm 20.12^{*} \\
184.8 \pm 26\end{array}$ & $\begin{array}{r}218.5 \pm 26.72^{*} \\
213.79 \pm 20.02^{*}\end{array}$ & $\begin{array}{l}229.24 \pm 24.14^{*} \\
242.99 \pm 29.84^{*}\end{array}$ & $\begin{array}{r}266.22 \pm 35.65^{*} \\
279.2 \pm 19.45^{*}\end{array}$ \\
\hline PR & $\begin{array}{l}\text { HF length } \\
\text { SK }\end{array}$ & $\begin{array}{l}197.37 \pm 18.36 \\
226.03 \pm 25.97\end{array}$ & $\begin{array}{l}255.75 \pm 28.73 \\
270.76 \pm 20.67\end{array}$ & $\begin{array}{l}281.02 \pm 32.28 \\
297.85 \pm 35.67\end{array}$ & $\begin{array}{r}696.49 \pm 23.75 \\
460.1 \pm 28.77\end{array}$ \\
\hline CTR & $\begin{array}{l}\text { HF length } \\
\text { SK }\end{array}$ & $\begin{array}{r}214 \pm 26.29 \\
239.37 \pm 20.71\end{array}$ & $\begin{array}{l}252.03 \pm 30.94 \\
285.85 \pm 19.27\end{array}$ & $\begin{array}{l}276.52 \pm 34.06 \\
293.16 \pm 31.07\end{array}$ & $\begin{array}{l}709.37 \pm 27.52 \\
419.35 \pm 26.05\end{array}$ \\
\hline
\end{tabular}

Values are presented in micrometers as means \pm SD. Total $n=54(n=18$ per group). SK $=$ Skin thickness. * Statistically significant difference compared to the CTR group $(\mathrm{p}<0.05)$.

\section{Results}

Signals from the Sympathetic Nervous System Promote Normal Hair Growth

To investigate whether the sympathetic nervous system participates in HF cycling, we used chemical sympathectomy and depilation models. We administered i.p. injections of 6-OHDA to mice (6-OHDA group) to selectively destroy sympathetic nerve fiber terminals without affecting the cell bodies, thus leading to a reduction in catecholamine content. To distinguish between the 2 catecholaminergic pathways, we administered PR to the drinking water of mice for 3 weeks (PR group) [Katayama et al., 2006]. The HF of 6- to 8-week-old mice are initially in the telogen stage and then begin to cycle through the stages, accompanied by changes in skin color (telogen, pink; anagen, gray, and catagen, black) [Slominski et al., 1994; Muller-Rover et al., 2001; Stenn and Paus, 2001].

Depilation of mice in the 6-OHDA, PR, and CTR groups induced a uniform pink skin color in all mice, indicative of the telogen stage (fig. 1a). On days 3 and 5 after depilation, we did not observe a change in dorsal skin color. After 7 days, 4 out of 21 mice (19.1\%) in the PR group and 6 out of 24 mice (25\%) in the CTR group were gray in color, whereas none of the mice in the 6-OHDA group had changed color ( $\mathrm{p}<0.05$; fig. $1 \mathrm{~b})$. On day 9 after depilation, only 2 out of 20 mice (10\%) in the 6-OHDA group exhibited macroscopic signs of the anagen cycle (skin darkening). Conversely, 11 out of 19 mice (57.9\%) in the PR group and 13 out of 22 mice (59.1\%) in the CTR group displayed a deep-gray or black skin color at that time ( $\mathrm{p}<0.05$; fig. $1 \mathrm{~b}$ ). The back skin of the remainder of the mice in all 3 groups remained pink, without evidence of de novo hair shaft formation. On day 11, we continued to find significant differences among the 3 groups (6OHDA, 33.3\%; PR, 76.5\%, and CTR, 78.9\%; p < 0.05; fig. 1b). On day 14, about two thirds of the 6-OHDAtreated mice displayed a gray dorsal skin color, whereas $82 \%$ of the PR-treated mice and $87 \%$ of the CTR mice demonstrated deep-gray or black skin (fig. 1a, b). There was no difference in the number of mice that showed skin color changes among the 3 groups, but mice in the 6-OHDA group were lighter in color than the blackskinned mice, which even exhibited hair growth on the dorsal skin surface in the PR and CTR groups (fig. 1a). The distribution of all mice among the 3 groups over time is listed in table 1.

Many methods for HF stage classification have been reported [Slominski et al., 1994; Muller-Rover et al., 2001]. HF length and skin thickness are among the easiest parameters for assessing stage. We performed H\&E staining to visualize $\mathrm{HF}$ and the skin and measure their length and thickness. On days 3, 5, 7, and 9 after depilation, the HF length and skin thickness of the mice in the PR and CTR groups were significantly greater compared to the values for mice in the 6-OHDA group ( $\mathrm{p}<0.05$; fig. 2 ; table 2). The increase in skin thickness was accompanied by thickening of the subcutis, but the subcutis of mice in the PR and CTR groups was thicker than the subcutis of mice in the 6-OHDA group. When HF are in the anagen stage, the bulb can reach deep into the subcutis [MullerRover et al., 2001]. On day 9, the formation of new hair shafts was apparent in the PR and CTR groups but not in the 6-OHDA group (fig. 2a). In addition, the DP appeared larger in the PR and CTR groups. Collectively, these results suggest that normal sympathetic nerve signals shorten the time between the telogen and anagen stages. 


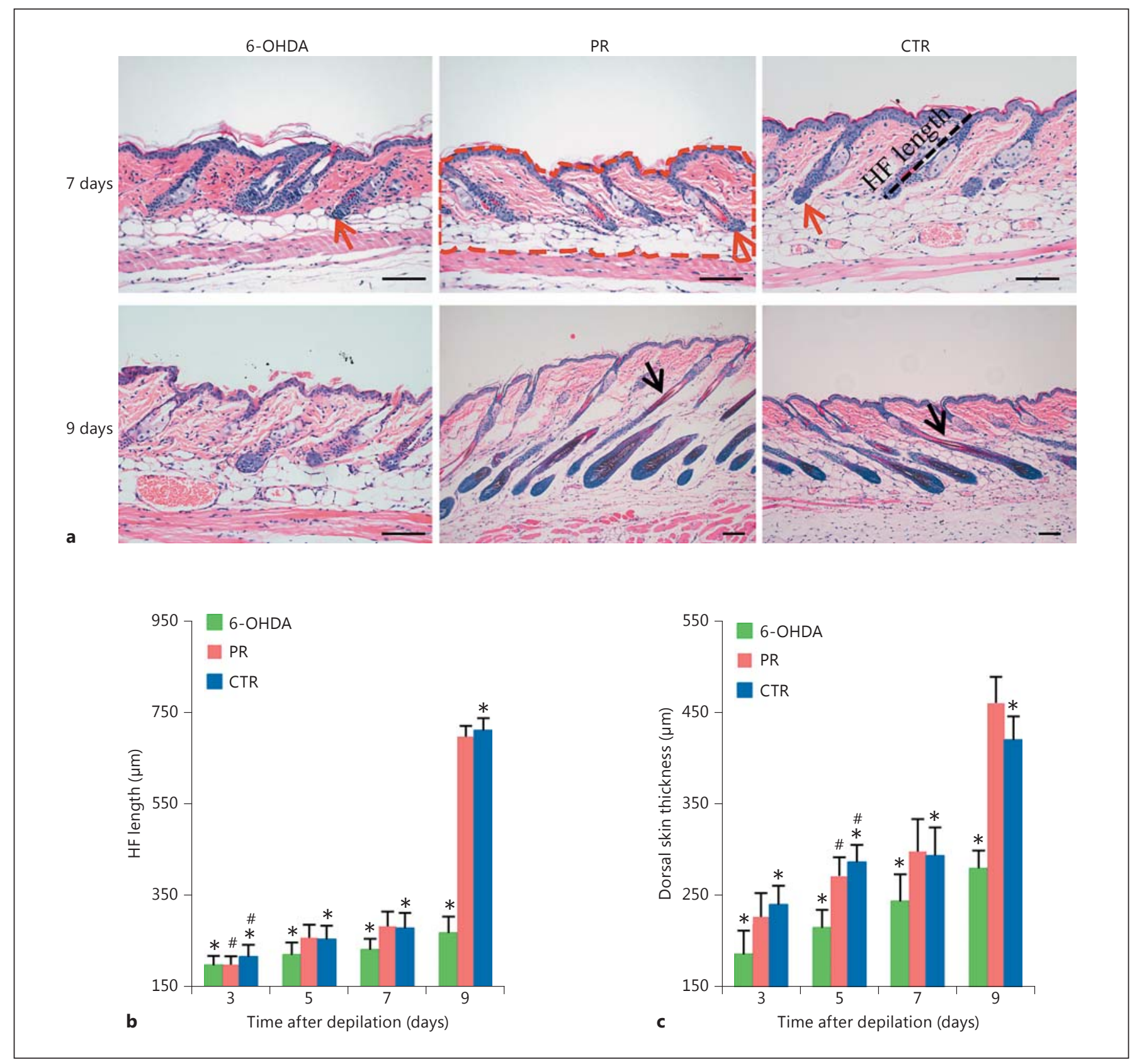

Fig. 2. Sympathetic nerves regulate HF length and skin thickness. a H\&E staining was performed on skin samples harvested 3, 5, 7, and 9 days after depilation. HF length and skin thickness were measured. The black dotted line represents the HF. The area within the red dotted line represents the skin area. The black arrows

The Sympathetic Nervous System Stimulates the Proliferation of Keratinocytes in the Epithelium and HF

We evaluated the proliferation of keratinocytes located in the back skin by BrdU incorporation. The number of BrdU-positive cells in the epidermal layer and HF in- indicate the hair shaft. The red arrows indicate the DP. Scale bar = $100 \mu \mathrm{m}$. b HF length is presented as the mean length of all photomicrographs \pm SD. c Skin thickness is presented as the mean thickness of all photomicrographs \pm SD. $\mathrm{n}=18 .{ }^{*} \mathrm{p}<0.05 .{ }^{\#} \mathrm{p}<0.05$ (PR vs. CTR group). 
Fig. 3. Sympathetic nerves regulate the proliferation of keratinocytes in HF. a BrdU immunohistochemistry was performed on skin samples harvested 3, 5, 7, and 9 days after depilation. More than 1,000 keratinocytes were counted. Scale bar $=100 \mu \mathrm{m}$. b Percentage of BrdU-positive cells in HF. c Percentage of BrdU-positive cells in the IFE. d Ki67 immunohistochemistry was performed on skin samples harvested 5 days after depilation. More than 1,000 keratinocytes were counted. Scale bar $=100$ $\mu \mathrm{m}$. e Percentage of Ki67-positive cells in HF. f Percentage of Ki67-positive cells in the IFE. ${ }^{*} \mathrm{p}<0.05$.

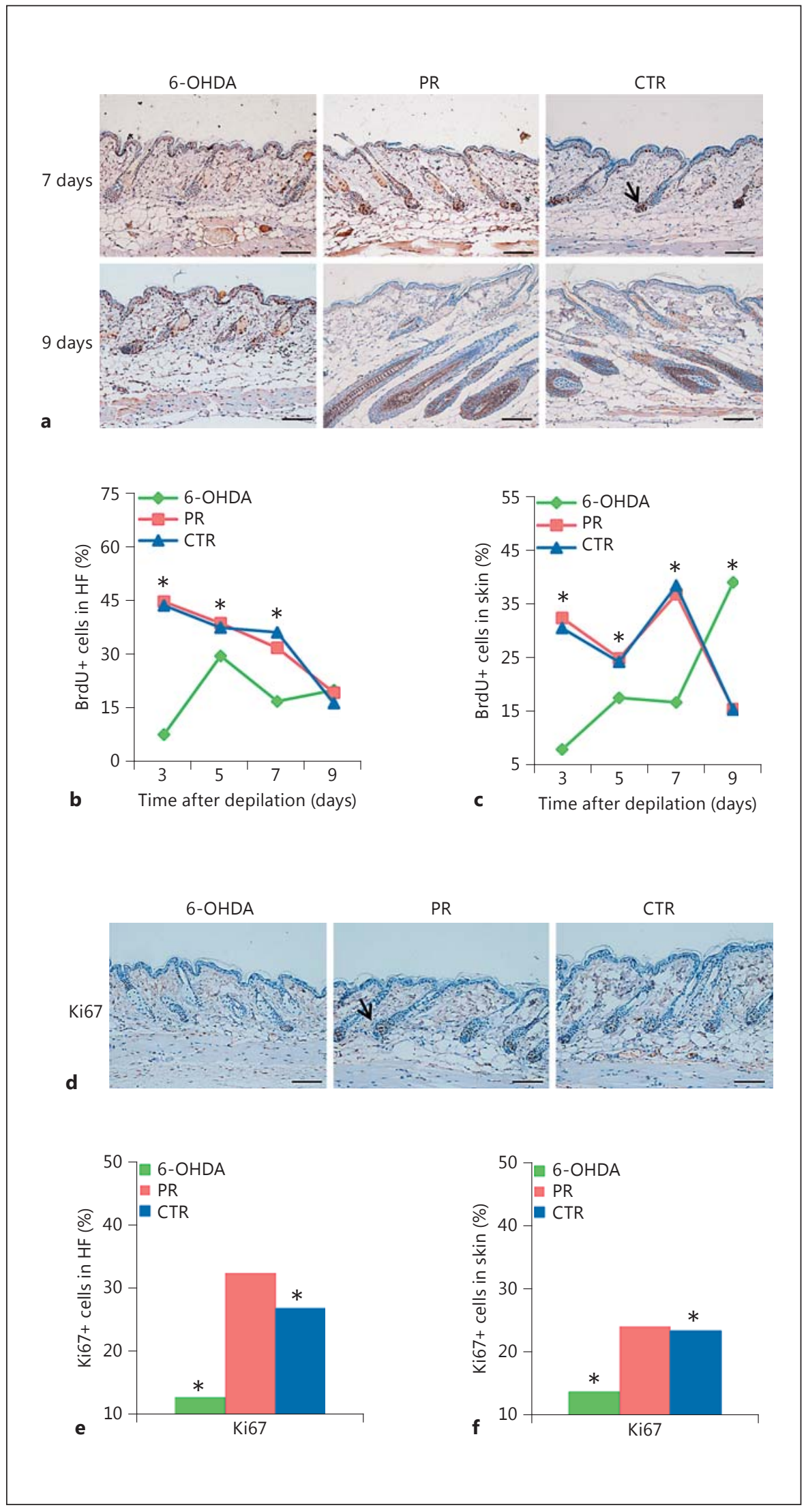


Table 3. BrdU-positive cells in the HF and epidermis over 9 days after depilation

\begin{tabular}{llrlll}
\hline \multicolumn{2}{c}{ Day: } & 3 & 5 & 7 & 9 \\
\hline 6-OHDA & HF & 78/1,003* & 297/1,001* & $200 / 1,176^{*}$ & $249 / 1,236^{*}$ \\
& EP & $106 / 1,291^{*}$ & $206 / 1,158^{*}$ & $336 / 1,053$ & $398 / 1,014^{*}$ \\
\hline PR & HF & $464 / 1,058$ & $473 / 1,219$ & $336 / 1,053$ & $292 / 1,504$ \\
& EP & $336 / 1,030$ & $253 / 1,007$ & $371 / 1,002$ & $160 / 1,020$ \\
\hline CTR & HF & $448 / 1,024$ & $408 / 1,086$ & $367 / 1,013$ & $196 / 1,190$ \\
& EP & $310 / 1,008$ & $247 / 1,009$ & $521 / 1,348$ & $158 / 1,013$ \\
\hline
\end{tabular}

Values are presented as numbers of BrdU-positive cells over the total number of cells. EP = Epidermis. ${ }^{*}$ Statistically significant difference compared to the CTR group ( $<0.05)$.

Table 4. Ki67-positive cells in the HF and epidermis 5 days after depilation

\begin{tabular}{lll}
\hline & HF & EP \\
\hline 6 -OHDA & $142 / 1,126^{*}$ & $145 / 1,056^{*}$ \\
PR & $344 / 1,064$ & $295 / 1,223$ \\
CTR & $348 / 1,304$ & $289 / 1,227$ \\
\hline
\end{tabular}

Values are presented as numbers of Ki67-positive cells over the total number of cells. EP = Epidermis. * Statistically significant difference compared to the CTR group $(\mathrm{p}<0.05)$.

Table 5. HF length and skin thickness in ex vivo skin cultures

\begin{tabular}{lcc}
\hline \multicolumn{2}{c}{ HF } & SK \\
\hline CTR & $76.01 \pm 18.42$ & $69.6 \pm 12.93$ \\
NE & $163.23 \pm 19.89^{*}$ & $120.61 \pm 16.2^{*}$ \\
PR & $140.78 \pm 18.94^{*}$ & $105.2 \pm 13.05^{*}$ \\
PH & $92.79 \pm 12.84$ & $82.22 \pm 16.22$
\end{tabular}

Vales are presented in micrometers as means \pm SD. Total $n=$ $72\left(\mathrm{n}=18\right.$ per group). SK = Skin thickness. ${ }^{*}$ Statistically significant difference compared to the CTR group ( $<<0.05)$.

thetic nervous system enhances the proliferation of $\mathrm{HF}$ and epidermal keratinocytes. However, on day 9, more BrdU-positive cells were detected in the 6-OHDA group instead of the PR and CTR groups ( $<<0.05$; fig. 3; table 3).

Ki67 is expressed in the nuclei of cells during different phases of the cell cycle, except in the G0 quiescent phase.
As such, Ki67 expression is routinely used as a measure of cell proliferation [Andre et al., 2015]. We used Ki67 staining to confirm our BrdU results. Five days after depilation, the number of Ki67-positive cells was consistent with the number of BrdU-positive cells in the HF for all groups (PR, 32\%; CTR, 26\%, and 6-OHDA, 13\%; $\mathrm{p}<0.05$; fig. 3; table 4).

\section{NE Promotes the Initiation of HF in Cultured Neonatal Skin}

The sympathetic neurotransmitter NE is released from the sympathetic nerve and regulates the biological activity primarily of $\alpha-A R$ and, to a lesser extent, of $\beta$-AR [Katayama et al., 2006]. Administration of 6-OHDA to mice induces a long-lasting chemical sympathectomy, resulting in the depletion of peripheral stores of NE [Gosain et al., 2006; Peters et al., 2007]. Previous studies have demonstrated NE depletions of approximately $95 \%$ in a variety of tissue beds following 6-OHDA treatment [Lorton et al., 1990; Kohm and Sanders, 1999; Gosain et al., 2006]. To further determine whether the sympathetic nervous system is involved in NE-mediated initiation of HF, HF formation in the dorsal skin of neonatal mice was examined in an organotypic skin culture model. The dorsal skins of neonatal littermates were cultured with NE or PBS for 3 days. To investigate which AR participate, skins cultured in the presence of NE were also pretreated with PR or phentolamine for 30 min each day. H\&E staining revealed that significantly thicker skin and longer HF were present in skin samples treated with NE and phentolamine compared to those treated with PBS or PR (fig. 4; table 5). Taken together, these results suggest that NE promotes the initiation of $\mathrm{HF}$ through $\alpha-\mathrm{AR}$

\section{Discussion}

Clinical and experimental evidence supports the involvement of sympathetic nerve fibers during hair growth. For example, the number of nerve fibers containing NE was increased during the early growth phase of the hair cycle in the dermis and the subcutis compared to the resting phase, declining again during the late anagen phase [Botchkarev et al., 1999]. Furthermore, a massive increase in the number of tyrosine hydroxylase-immunoreactive dermal nerve fibers accompanied by an increase in NE content was observed particularly in outer root sheath keratinocytes [Botchkarev et al., 1999]. The $\beta 2$-AR agonist isoproterenol also accelerated HF growth in organotypic skin culture [Peters et al., 2007]. 


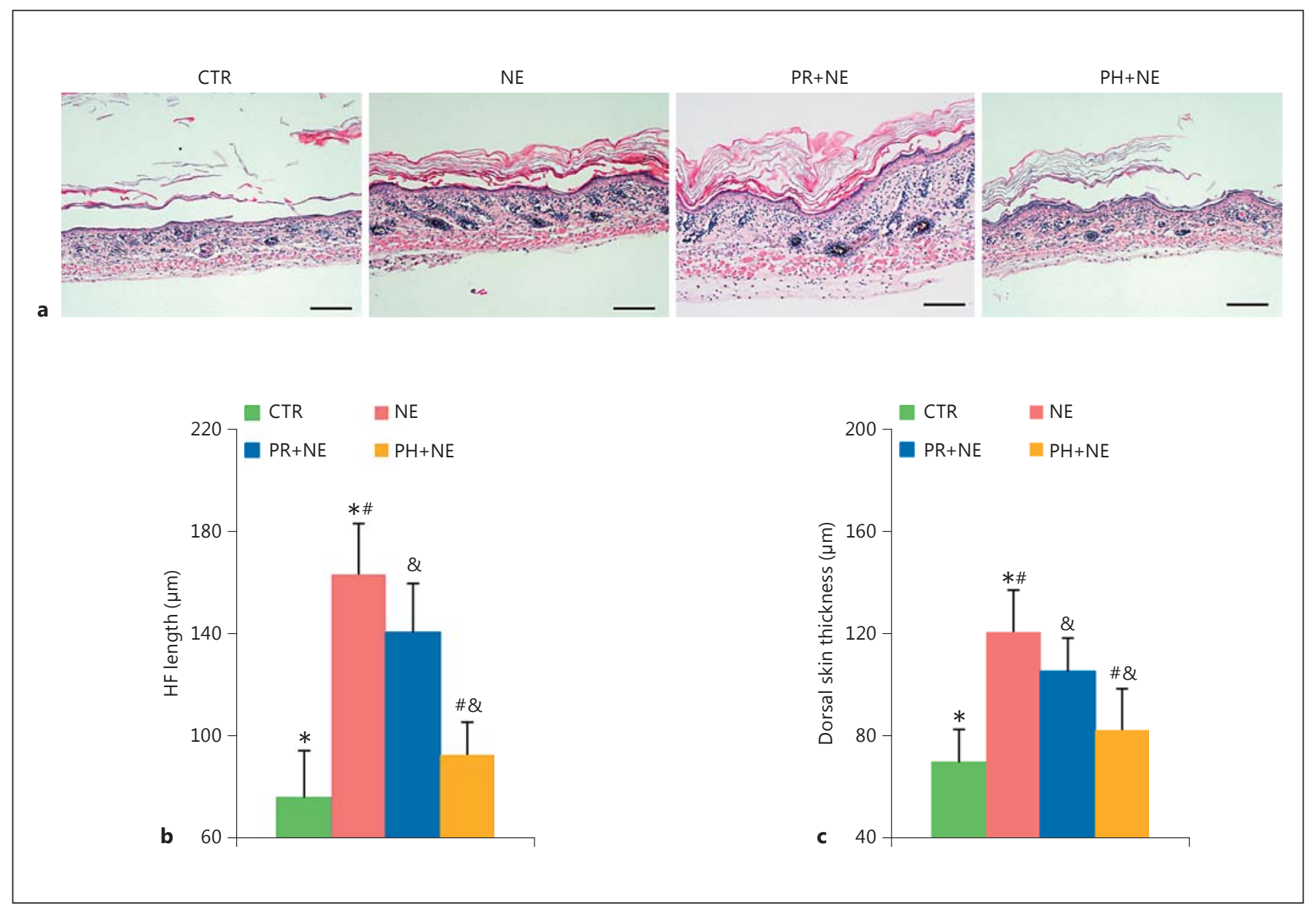

Fig. 4. NE promotes the initiation of HF in neonatal mouse skin cultures. a H\&E staining was performed on organotypic skin cultures treated with CTR, NE, PR, or phentolamine (PH) for 3 days. HF length and skin thickness were measured. Scale bar $=100 \mu \mathrm{m}$. b HF length is presented as the mean length of all photomicrographs \pm SD. c Skin thickness is presented as the mean thickness of all photomicrographs \pm SD. $n=18 .{ }^{*} \mathrm{p}<0.05 .{ }^{*} \mathrm{p}<0.05$ (NE vs. $\mathrm{PH}+\mathrm{NE}$ group). ${ }^{\&} \mathrm{p}<0.05$ ( $\mathrm{PH}+\mathrm{NE}$ vs. $\mathrm{PR}+\mathrm{NE}$ group).
Asada-Kubota [1995] further demonstrated that subcutaneous injections of 6-OHDA inhibited hair growth in neonatal mice. In the present study, we also found that chemical sympathectomy inhibited hair growth in adult C57BL/6 mice, lending further support to the concept that the sympathetic nervous system controls hair growth. Conversely, Peters et al. [2007] reported that subcutaneous injections of 6-OHDA induced a premature onset of anagen in the lower-back skin of C57BL/6 mice. We suspect that neurotransmitters play biological roles in concentration-dependent manners. For example, $750 \mathrm{nM}$ NE significantly promoted corneal epithelial cell proliferation, whereas $100 \mu \mathrm{M}$ NE inhibited proliferation and $10 \mathrm{mM}$ was toxic [Murphy et al., 1998; Grando et al., 2006]. Similar findings have been demon- strated for dopamine [Parrado et al., 2012; Langan et al., 2013]. In the study by Peters et al. [2007], 6-OHDA (0.03 $\mathrm{mg} / \mathrm{g}$ of body weight) was injected 1 time on the first and second days to selectively destroy NE-containing nerve endings. NE levels in the spleen decrease by about $95 \%$ immediately following 6-OHDA exposure and return to control levels within 56 days after exposure [Lorton et al., 1990; Kruszewska et al., 1995; Kohm and Sanders, 1999]. Thus, the NE levels in the mice of Peters et al. [2007] recovered within 20 days after exposure to 6-OHDA. However, we injected 6-OHDA every 2 days over 3 weeks. The NE levels began to recover on the last day of 6-OHDA administration. The different rates of NE recovery may account for the discrepancies between the two outcomes. 
Perpetual cycling of HF is coupled with macroscopic signs of skin color changes. Our findings show that depletion of NE stores from sympathetic nerve terminals via i.p. injection of 6-OHDA induced gradual hair growth on the back. Nine days after depilation-induced follicle growth, over half of the PR-treated and CTR mice exhibited a gray or black dorsal-skin color. In contrast, only $10 \%$ of the 6 -OHDA-treated mice were gray, suggesting that NE may regulate hair growth.

HF present in different areas of the body produce hairs of different lengths, which are proportional to the duration of the anagen cycle [Danilenko et al., 1996; MullerRover et al., 2001]. For example, scalp HF remain in the anagen stage for 2-8 years to produce long hair, whereas eyebrow HF enter the anagen stage for only $2-3$ months [Stenn and Paus, 2001]. We found that HF in PR-treated and CTR mice were significantly longer than those in 6-OHDA-treated mice. On days 3, 5, 7, and 9 after depilation, the skin was also obviously thicker, further suggesting that NE is a major determinant of hair growth. Kim et al. [2013] found that collagen synthesis increased in thicker skin during the anagen stage, suggesting that collagen promotes, and may even initiate, hair growth. However, determining the mechanisms that orchestrate HF transitions between these 3 stages remains one of the key challenges in hair research.

The HF develops from the embryonic epidermis as an epithelial finger [Fusenig et al., 1994; Botchkarev and Fessing, 2005; Zhang et al., 2008]. The balance between proliferation and apoptosis of keratinocytes in the HF maintains the regenerative capacity of hair [Muller-Rover et al., 2001; Bollag, 2012]. It has been previously reported that NE stimulates keratinocyte proliferation in vitro [Murphy et al., 1998]. Additionally, there is in vivo evidence for sympathetic stimulation of corneal epithelial proliferation [Reidy et al., 1994; Kim et al., 2014]. In this study, the number of proliferating cells, as marked by BrdU or Ki67 positivity, was significantly reduced in sympathectomized animals compared to PR-treated or CTR mice, which could have directly influenced the slower hair growth observed in the 6-OHDA group. When the $\mathrm{HF}$ is in the late stages of the anagen cycle, keratinocyte proliferation decreases [Muller-Rover et al., 2001]. Therefore, more proliferating keratinocytes were observed in 6-OHDA-treated mice 9 days after depilation. Keratinocytes secrete many different growth factors, such as neurotrophin, fibroblast growth factor (FGF)-1, FGF-2, FGF10, keratinocyte growth factor, and platelet-derived growth factor, all of which have been shown to promote hair growth [Guo et al., 1996; Grewe et al., 2000; Botch- karev et al., 2006; Barrientos et al., 2008; Lin et al., 2015]. We also found that increased keratinocyte proliferation in PR-treated and CTR mice results in more cells in the skin (data not shown). Further studies to identify how growth factors secreted by keratinocytes promote hair growth are necessary.

HF contain quiescent SC located in a structure termed the bulge (Bu-SC), and more activation-prone SC are anatomically located immediately below the Bu-SC in the hair germ (HG) (fig. 5a). Activated SC in the HG proliferate and differentiate into transit-amplifying cells (TAC) that form a matrix structure and eventually give rise to the differentiated cells of the HF (fig. 5b). SC in the HF have been demonstrated to be regulated by numerous cellular sources, most prominently by cells located in the DP. Activated $\mathrm{SC}$ in the $\mathrm{HG}$ receive proliferative signals, possibly from the DP, and differentiate into TAC [Kalaitzidis and Scadden, 2014]. Hsu et al. [2014] showed that signals emanating from TAC directly stimulate the proliferation of $\mathrm{Bu}-\mathrm{SC}$ as well as the DP, creating a positive feedback loop for TAC production and facilitating proper HF regeneration. In this study, we observed greater numbers of cells in the TAC area of PR-treated and CTR mice than in sympathectomized animals (fig. 5c). Thus, TAC may act as effectors of sympathetic nerve signals to regulate hair growth, but further studies are needed to confirm our hypothesis.

A recent study reported that topical application of the $a$-adrenergic vasoconstrictors epinephrine or NE to the skin of neonatal rats before a considerable radiation or chemotherapeutic insult prevented up to $100 \%$ of the alopecia observed in CTR animals [Soref and Fahl, 2015]. Chemotherapy-induced alopecia may be attributed to peripheral neurotoxic effects [Cavaletti et al., 2015; Cavaletti and Marmiroli, 2015; Dietrich et al., 2015], thus explaining why application of epinephrine or NE suppressed alopecia. We found that hair growth was inhibited in sympathectomized animals and hair growth proceeded in animals with a normal sympathetic nervous system, suggesting that normal hair growth requires NE, consistent with past findings. Our finding that NE stimulated HF progression in an organotypic skin culture model further confirmed the effect of NE. Our finding that NE stimulated HF progression in an organotypic skin culture model is consistent with the inhibited hair growth observed in sympathectomized animals. The organization and function of human and murine HF are similar - both contain the same principal cell types and undergo repetitive cycling that alternates between the anagen, catagen, and telogen phases [Oh et al., 2016]. However, significant 


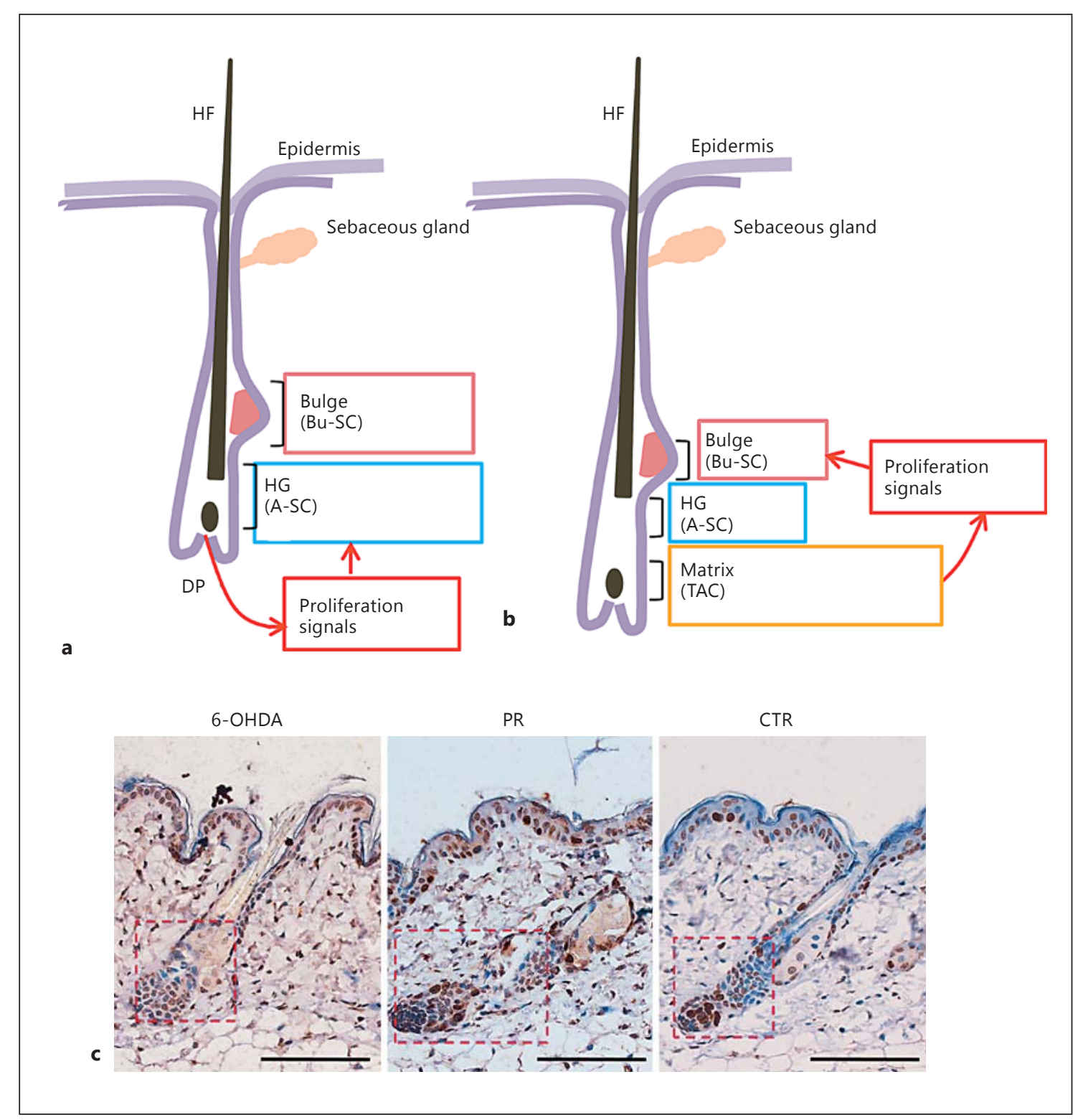

Fig. 5. a During HF regeneration, signals from the DP evoke proliferative responses in activated SC (A-SC) located in the HG below a layer of quiescent $\mathrm{Bu}-\mathrm{SC}$, leading to the initiation of $\mathrm{HF}$ growth. b HG-SC proliferation leads to the production of TAC and downward expansion of the HF area. Signals emanating from TAC di- rectly stimulate $\mathrm{Bu}-\mathrm{SC}$ and DP proliferation, which feeds back on TAC production, resulting in proper HF regeneration. c BrdU staining was performed on skin samples from mice treated with 6-OHDA or PR or CTR animals. The dotted lines indicate the area of TAC in a single HF. Scale bars $=100 \mu \mathrm{m}$. interspecies differences exist. The recent establishment of 3-D spheroid culture of DP cells has provided an attractive model in which to investigate human hair without the limitations of human sampling [Higgins et al., 2013].

In summary, this study provides suggestive evidence that sympathetic nerves regulate keratinocyte behaviors to promote hair growth. The underlying mechanisms and signaling pathways are complicated and require further investigation. However, our findings offer a new perspective for understanding stress-related, chemotherapy-, and radiotherapy-induced alopecia. Furthermore, targeted pharmacologic manipulations of adrenergic signaling pathways in the skin may represent novel strategies for modulating hair growth in health and disease. 


\section{Acknowledgements}

This work was supported by the National Natural Science Foundation of China (81171812 and 81272105), the National Basic Science and Development Program (973 Program, 2012CB518105), Health and Medical Treatment Collaborative Innovation Major Special Projects of Guangzhou (201508020253), and the Science and Technology Key Project of Guangdong Province (2014B020212010).

\section{References}

Andre, F., M. Arnedos, A. Goubar, A. Ghouadni, S. Delaloge (2015) Ki67 - no evidence for its use in node-positive breast cancer. Nat Rev Clin Oncol 12: 296-301.

Arck, P.C., A. Slominski, T.C. Theoharides, E.M. Peters, R. Paus (2006) Neuroimmunology of stress: skin takes center stage. J Invest Dermatol 126: 1697-1704.

-Asada-Kubota, M. (1995) Inhibition of hair growth by subcutaneous injection of a sympathetic neurotoxin, 6-hydroxydopamine in neonatal mice. Anat Embryol 191: 407-414.

- Barrientos, S., O. Stojadinovic, M.S. Golinko, H. Brem, M. Tomic-Canic (2008) Growth factors and cytokines in wound healing. Wound Repair Regen 16: 585-601.

Blanpain, C., E. Fuchs (2009) Epidermal homeostasis: a balancing act of stem cells in the skin. Nat Rev Mol Cell Biol 10: 207-217.

Bollag, W.B. (2012) Mediator1: an important intermediary of vitamin $\mathrm{D}$ receptor-regulated epidermal function and hair follicle biology. J Invest Dermatol 132: 1068-1070.

Botchkarev, V.A., M.Y. Fessing (2005) Edar signaling in the control of hair follicle development. J Investig Dermatol Symp Proc 10: 247251.

Botchkarev, V.A., E.M. Peters, N.V. Botchkareva, M. Maurer, R. Paus (1999) Hair cycle-dependent changes in adrenergic skin innervation, and hair growth modulation by adrenergic drugs. J Invest Dermatol 113: 878-887.

Botchkarev, V.A., M. Yaar, E.M. Peters, S.P. Raychaudhuri, N.V. Botchkareva, A. Marconi, S.K. Raychaudhuri, R. Paus, C. Pincelli (2006) Neurotrophins in skin biology and pathology. J Invest Dermatol 126: 1719-1727.

Cavaletti, G., P. Alberti, P. Marmiroli (2015) Chemotherapy-induced peripheral neurotoxicity in cancer survivors: an underdiagnosed clinical entity? Am Soc Clin Oncol Educ Book 35: e553-e560.

Cavaletti, G., P. Marmiroli (2015) Chemotherapy-induced peripheral neurotoxicity. Curr Opin Neurol 28: 500-507.

-Cerqueira, M.T., A.M. Frias, R.L. Reis, A.P Marques (2014) Boosting and rescuing epidermal superior population from fresh keratinocyte cultures. Stem Cell Dev 23: 34-43.

Danilenko, D.M., B.D. Ring, G.F. Pierce (1996) Growth factors and cytokines in hair follicle development and cycling: recent insights from animal models and the potentials for clinical therapy. Mol Med Today 2: 460-467.

Dietrich, J., M. Prust, J. Kaiser (2015) Chemotherapy, cognitive impairment and hippocampal toxicity. Neuroscience 19: 224-232.
Fusenig, N.E., A. Limat, H.J. Stark, D. Breitkreutz (1994) Modulation of the differentiated phenotype of keratinocytes of the hair follicle and from epidermis. J Dermatol Sci 7: S142-S151.

Glaser, R., J.K. Kiecolt-Glaser (2005) Stress-induced immune dysfunction: implications for health. Nat Rev Immunol 5: 243-251.

Gosain, A., S.B. Jones, R. Shankar, R.L. Gamelli, L.A. DiPietro (2006) Norepinephrine modulates the inflammatory and proliferative phases of wound healing. J Trauma 60: 736-744.

Grando, S.A., M.R. Pittelkow, K.U. Schallreuter (2006) Adrenergic and cholinergic control in the biology of epidermis: physiological and clinical significance. J Invest Dermatol 126: 1948-1965.

Grewe, M., K. Vogelsang, T. Ruzicka, H. Stege, J. Krutmann (2000) Neurotrophin-4 production by human epidermal keratinocytes: increased expression in atopic dermatitis. J Invest Dermatol 114: 1108-1112.

Guo, L., L. Degenstein, E. Fuchs (1996) Keratinocyte growth factor is required for hair development but not for wound healing. Genes Dev 10: 165-175.

Hansen, L.S., J.E. Coggle, J. Wells, M.W. Charles (1984) The influence of the hair cycle on the thickness of mouse skin. Anat Rec 210: 569573.

Heidt, T., H.B. Sager, G. Courties, P. Dutta, Y. Iwamoto, A. Zaltsman, C. von Zur Muhlen, C. Bode, G.L. Fricchione, J. Denninger, C.P. Lin, C. Vinegoni, P. Libby, F.K. Swirski, R. Weissleder, M. Nahrendorf (2014) Chronic variable stress activates hematopoietic stem cells. Nat Med 20: 754-758.

Higgins, C.A., J.C. Chen, J.E. Cerise, C.A.B. Jahoda, A.M. Christiano (2013) Microenvironmental reprogramming by three-dimensional culture enables dermal papilla cells to induce de novo human hair-follicle growth. Proc Natl Acad Sci USA 110: 19679-19688.

Hsu, Y.C., L. Li, E. Fuchs (2014) Transit-amplifying cells orchestrate stem cell activity and tissue regeneration. Cell 157: 935-949.

Ito, T. (2010) Hair follicle is a target of stress hormone and autoimmune reactions. J Dermatol Sci 60: 67-73.

-Jia, Q., M. Zhang, Y. Kong, S. Chen, Y. Chen, X. Wang, L. Zhang, W. Lang, L. Zhang, L. Zhang (2013) Activin B promotes initiation and development of hair follicles in mice. Cells Tissues Organs 198: 318-326.

Kalaitzidis, D., D.T. Scadden (2014) Tic-TACs: refreshing hair growth. Cell 157: 769-770.

Katayama, Y., M. Battista, W.M. Kao, A. Hidalgo, A.J. Peired, S.A. Thomas, P.S. Frenette (2006)
Signals from the sympathetic nervous system regulate hematopoietic stem cell egress from bone marrow. Cell 124: 407-421.

Kim, C., D. Barbut, M.H. Heinemann, G. Pasternak, M.I. Rosenblatt (2014) Synthetic neurotensin analogues are nontoxic analgesics for the rabbit cornea. Invest Ophthalmol Vis Sci 55: 3586-3593.

Kim, H.M., Y.Y. Lim, M.Y. Kim, I.P. Son, D.H. Kim, S.R. Park, S.K. Seo, M.S. Lee, S.K. Mun, C.W. Kim, B.J. Kim (2013) Inhibitory effect of tianeptine on catagen induction in alopecia areata-like lesions induced by ultrasonic wave stress in mice. Clin Exp Dermatol 38: 758767.

Kohm, A.P., V.M. Sanders (1999) Suppression of antigen-specific Th2 cell-dependent IgM and IgG1 production following norepinephrine depletion in vivo. J Immunol 162: 5299-5308.

Kruszewska, B., S.Y. Felten, J.A. Moynihan (1995) Alterations in cytokine and antibody production following chemical sympathectomy in two strains of mice. J Immunol 155: 46134620.

Langan, E.A., E. Lisztes, T. Biro, W. Funk, J.E. Kloepper, C.E. Griffiths, R. Paus (2013) Dopamine is a novel, direct inducer of catagen in human scalp hair follicles in vitro. Br J Dermatol 168: 520-525.

Liang, Y., K.A. Silva, V. Kennedy, J.P. Sundberg (2011) Comparisons of mouse models for hair follicle reconstitution. Exp Dermatol 20: 1011-1015.

Lin, W.H., L.J. Xiang, H.X. Shi, J. Zhang, L.P. Jiang, P.T. Cai, Z.L. Lin, B.B. Lin, Y. Huang, H.L. Zhang, X.B. Fu, D.J. Guo, X.K. Li, X.J. Wang, J. Xiao (2015) Fibroblast growth factors stimulate hair growth through $\beta$-catenin and Shh expression in C57BL/6 mice. Biomed Res Int 2015: 1-9.

Lorton, D., D. Hewitt, D.L. Bellinger, S.Y. Felten, D.L. Felten (1990) Noradrenergic reinnervation of the rat spleen following chemical sympathectomy with 6-hydroxydopamine: pattern and time course of reinnervation. Brain Behav Immun 4: 198-222.

Miyata, S., Y. Oda, C. Matsuo, H. Kumura, K. Kobayashi (2014) Stimulatory effect of Brazilian propolis on hair growth through proliferation of keratinocytes in mice. J Agric Food Chem 62: 11854-11861.

Muller-Rover, S., B. Handjiski, C. van der Veen, S. Eichmuller, K. Foitzik, I.A. McKay, K.S. Stenn, R. Paus (2001) A comprehensive guide for the accurate classification of murine hair follicles in distinct hair cycle stages. J Invest Dermatol 117: 3-15. 
Murphy, C.J., S. Campbell, K. Araki-Sasaki, C.F. Marfurt (1998) Effect of norepinephrine on proliferation, migration, and adhesion of SV40 transformed human corneal epithelial cells. Cornea 17: 529-536.

Obeid, G., J. Helou, I. Maatouk, R. Moutran, R. Tomb (2013) Depilatory laser: a potential causative factor for inguinal hyperhidrosis: report of three cases. J Cosmet Laser Ther 15: 286-289.

Oh, J.W., J. Kloepper, E.A. Langan, Y. Kim, J. Yeo, M.J. Kim, T.C. Hsi, C. Rose, G.S. Yoon, S.J. Lee, J. Seykora, J.C. Kim, Y.K. Sung, M. Kim, R. Paus, M.V. Plikus (2016) A guide to studying human hair follicle cycling in vivo. J Invest Dermatol 136: 34-44.

Ouji, Y., M. Yoshikawa, A. Shiroi, S. Ishizaka (2006) Promotion of hair follicle development and trichogenesis by Wnt-10b in cultured embryonic skin and in reconstituted skin. Biochem Biophys Res Commun 345: 581-587.

-Parrado, A.C., A. Canellada, T. Gentile, E.B. ReyRoldan (2012) Dopamine agonists upregulate IL-6 and IL-8 production in human keratinocytes. Neuroimmunomodulation 19: 359366.

Peng, Y., S. Huang, Y. Wu, B. Cheng, X. Nie, H. Liu, K. Ma, J. Zhou, D. Gao, C. Feng, S. Yang, X. Fu (2013) Platelet rich plasma clot releasate preconditioning induced $\mathrm{PI} 3 \mathrm{~K} / \mathrm{AKT} / \mathrm{NF} \mathrm{KB}$ signaling enhances survival and regenerative function of rat bone marrow mesenchymal stem cells in hostile microenvironments. Stem Cells Dev 22: 3236-3251.

Peters, E.M., P.C. Arck, R. Paus (2006) Hair growth inhibition by psychoemotional stress: a mouse model for neural mechanisms in hair growth control. Exp Dermatol 15: 1-13.

Peters, E.M.J., M. Maurer, V.A. Botchkarev, D.S. Gordon, R. Paus (2007) Hair growth-modulation by adrenergic drugs. Exp Dermatol 8: 274-281.
Reidy, J.J., J. Zarzour, H.W. Thompson, R.W. Beuerman (1994) Effect of topical beta blockers on corneal epithelial wound healing in the rabbit. Br J Ophthalmol 78: 377-380.

-Semenova, E., H. Koegel, S. Hasse, J.E. Klatte, E. Slonimsky, D. Bilbao, R. Paus, S. Werner, N. Rosenthal (2008) Overexpression of mIGF-1 in keratinocytes improves wound healing and accelerates hair follicle formation and cycling in mice. Am J Pathol 173: 1295-1310.

Slominski, A. (2007) A nervous breakdown in the skin: stress and the epidermal barrier. J Clin Invest 117: 3166-3169.

Slominski, A., N.V. Botchkareva, V.A. Botchkarev, A. Chakraborty, T. Luger, M. Uenalan, R. Paus (1998) Hair cycle-dependent production of ACTH in mouse skin. Biochim Biophys Acta 1448: 147-152.

Slominski, A., R. Paus, P. Plonka, A. Chakraborty, M. Maurer, D. Pruski, S. Lukiewicz (1994) Melanogenesis during the anagen-catagentelogen transformation of the murine hair cycle. J Invest Dermatol 102: 862-869.

Slominski, A., P.M. Plonka, A. Pisarchik, J.L. Smart, V. Tolle, J. Wortsman, M.J. Low (2005) Preservation of eumelanin hair pigmentation in proopiomelanocortin-deficient mice on a nonagouti $(\mathrm{a} / \mathrm{a})$ genetic background. Endocrinology 146: 1245-1253.

Slominski, A., J. Wortsman (2000) Neuroendocrinology of the skin. Endocr Rev 21: 457487.

Slominski, A., J. Wortsman, T. Luger, R. Paus, S. Solomon (2000) Corticotropin releasing hormone and proopiomelanocortin involvement in the cutaneous response to stress. Physiol Rev 80: 979-1020.

Slominski, A., J. Wortsman, R.C. Tuckey, R. Paus (2007) Differential expression of HPA axis homolog in the skin. Mol Cell Endocrinol 265-266: 143-149.
Slominski, A.T., M.A. Zmijewski, C. Skobowiat, B. Zbytek, R.M. Slominski, J.D. Steketee (2012) Sensing the environment: regulation of local and global homeostasis by the skin's neuroendocrine system. Adv Anat Embryol Cell Biol 212: v, vii, 1-115.

Soref, C.M., W.E. Fahl (2015) A new strategy to prevent chemotherapy and radiotherapy-induced alopecia using topically applied vasoconstrictor. Int J Cancer 136: 195-203.

Stener-Victorin, E., K. Ploj, B.M. Larsson, A. Holmang (2005) Rats with steroid-induced polycystic ovaries develop hypertension and increased sympathetic nervous system activity. Reprod Biol Endocrinol 3: 44.

Stenn, K.S., R. Paus (2001) Controls of hair follicle cycling. Physiol Rev 81: 449-494.

Trueb, R.M. (2010) Systematic approach to hair loss in women. J Dtsch Dermatol Ges 8: 284 297, 284-298.

-Wygledowska-Kania, M., T. Bogdanowski (1996) Psychic factors in case histories of patients with alopecia areata - preliminary report (in Polish). Psychiatr Pol 30: 669-676.

Yang, E.V., A.K. Sood, M. Chen, Y. Li, T.D. Eubank, C.B. Marsh, S. Jewell, N.A. Flavahan, C. Morrison, P.E. Yeh, S. Lemeshow, R. Glaser (2006) Norepinephrine up-regulates the expression of vascular endothelial growth factor, matrix metalloproteinase (MMP)-2, and MMP-9 in nasopharyngeal carcinoma tumor cells. Cancer Res 66: 10357-10364.

Zhang, Y., T. Andl, S.H. Yang, M. Teta, F. Liu, J.T. Seykora, J.W. Tobias, S. Piccolo, R. SchmidtUllrich, A. Nagy, M.M. Taketo, A.A. Dlugosz, S.E. Millar (2008) Activation of beta-catenin signaling programs embryonic epidermis to hair follicle fate. Development 135: 21612172.

Zhang, M., N.Y. Liu, X.E. Wang, Y.H. Chen, Q.L. Li, K.R. Lu, L. Sun, Q. Jia, L. Zhang, L. Zhang (2011) Activin B promotes epithelial wound healing in vivo through RhoA-JNK signaling pathway. PLoS One 6: e25143. 